\title{
SOME RESULTS ON LIE $p$-ALGEBRAS
}

\author{
BY GEORGE B. SELIGMAN ${ }^{1}$
}

Communicated February 14, 1967

Let $\&$ be a Lie $p$-algebra ("restricted Lie algebra") over the field $\mathfrak{F}$ of prime characteristic $p\left[3\right.$, Chapter V]. Denote by $x^{p}$ the image of $x \in L$ under the $p$-power operation, by $x^{p^{k}}$ the image of $x$ under the $k$ th iterate of $x \rightarrow x^{p}$, with $x^{p^{0}}=x$. Let $\langle x\rangle$ be the subalgebra of $\mathfrak{R}$ generated by $x$, i.e., the space of linear combinations of the $x^{p^{k}}$, $k=0,1,2, \cdots$. Call $x \in \mathfrak{R}$ separable if $x \in\left\langle x^{p}\right\rangle$, nilpotent if $x^{p^{k}}=0$ for some $k$. Then we have proved the following decomposition theorem, which yields a slightly sharpened form of the Jordan-Chevalley decomposition $[2$, p. 71$]$ for linear transformations in the case of prime chtracteristic.

TheOREM 1. Let $x \in R$, a Lie p-algebra of finite dimension over the perfect field $\mathfrak{F}$. Then there exist elements $s, n \in\langle x\rangle$ with $s$ separable and $n$ nilpotent, such that $x=s+n$. If $y \in \mathfrak{R}$ is separable, $z \in \mathbb{R}$ nilpotent, $[y z]=0$, and $x=y+z$, then $y=s$ and $z=n$.

A subalgebra $\mathfrak{T}$ of the Lie $p$-algebra $\mathbb{R}$ is called toral if $\mathfrak{T}$ is commutative and if every element of $\mathfrak{I}$ is separable. A subalgebra $\mathfrak{N}$ is called $n i l$ if every element of $\mathfrak{R}$ is nilpotent. For a Lie $p$-algebra $\&$ of endomorphisms of a finite-dimensional vector space over an algebraically closed field, to say that $\mathbb{R}$ is triangulable is to say that [RR] is nil. In this connection we have the following result.

TheOREM 2. Let $\mathbb{R}$ be a Lie p-algebra over the perfect field $\mathfrak{F}$, and suppose that [RR] is nil. Let $\mathfrak{R}$ be the set of nilpotent elements of $\mathfrak{R}$, and let $\mathfrak{T}$ be any maximal toral subalgebra of $\mathfrak{R}$. Then $\mathfrak{R}$ is an ideal in $\mathfrak{R}$, and $\mathfrak{R}=\mathfrak{I}+\mathfrak{R}$. If , moreover, $\mathfrak{R}$ is nilpotent (as ordinary Lie algebra), then $\mathfrak{T}$ is the set of all separable elements of $\mathbb{R}$ and $\mathfrak{I}$ is central in $\mathfrak{R}$.

As to conjugacy of maximal toral subalgebras under these conditions we have shown the following:

THEOREM 3. Let $\mathfrak{R}$ be a Lie p-algebra over the field $\mathfrak{F}$. Suppose that the set $\mathfrak{R}$ of nilpotent elements is an ideal in $R$, and let $\mathfrak{T}_{1}$ and $\mathfrak{T}_{2}$ be toral subalgebras such that $\mathfrak{I}_{i}+\mathfrak{N}=\mathfrak{R}$. If $\mathfrak{N}$ is commutative, then there is an automorphism $\sigma$ of the Lie p-algebra $\mathfrak{R}$ such that $x^{\sigma}=x$ for all $x \in \mathfrak{R}$, with $y^{\sigma}-y \in \mathfrak{N}$ for all $y \in \mathfrak{R}$, and with $\mathfrak{T}_{1}^{\sigma}=\mathfrak{T}_{2}$. In general, there is no

${ }^{1}$ Research supported in part by grants NSF-GP-4017 and NSF-GP-6558, and by a Yale University Senior Faculty Fellowship. 
automorphism of the Lie p-algebra $\mathfrak{\Omega}$ mapping $\mathfrak{I}_{1}$ on $\mathfrak{I}_{2}$, even when $\mathfrak{F}$ is algebraically closed and $[\mathfrak{R}[\mathfrak{N} \mathfrak{N}]]=0$.

Over perfect fields in general, maximal toral subalgebras are related to Cartan subalgebras by the following:

THEOREM 4. Let $\mathfrak{\Omega}$ be a Lie p-algebra over a perfect field $\mathfrak{F}$. Let $\mathfrak{I}$ be a maximal toral subalgebra of $\mathfrak{R}, \quad \mathfrak{S}=N(\mathfrak{T})$ the normalizer of $\mathfrak{T}$. Then $\mathfrak{S}$ is a Cartan subalgebra of $\mathfrak{R}$. Conversely, if $\mathfrak{S}$ is a Cartan subalgebra of $\mathfrak{R}$, then $\mathfrak{S}=N(\mathfrak{T})$, where $\mathfrak{I}$ is the set of separable elements of $\mathfrak{S}$, and $\mathfrak{T}$ is a maximal toral subalgebra of $\mathbb{R}$.

THEOREM 5. Let $\mathbb{\Omega}$ be a Lie p-algebra over a perfect field $\mathfrak{F}$. Let $(x, y)$ be a nondegenerate symmetric associative (i.e., $([x y], z)=(x,[y z]))$ bilinear form on \& such that $(x, y)=0$ whenever $y$ is nilpotent and $[x y]$ $=0$. Then the Cartan subalgebras of $\mathbb{R}$ are the maximal toral subalgebras.

From Theorem 4 and the usual proof for infinite fields [3] we see that all Lie $p$-algebras possess Cartan subalgebras. The conditions of Theorem 5 are satisfied by trace forms of $p$-representations whenever such forms are nondegenerate, and also by the usual "quotient trace form" [6] on the $p r$ by $p r \varsubsetneqq$-matrices of trace zero, modulo scalars.

Our further results concern toral algebras. If $\Omega$ is any field of characteristic $p$ then $\Omega$, with its natural $p$-power, is a one-dimensional toral algebra over $\Re$, and may be regarded as a Lie $p$-algebra over any subfield of $\Omega$. In this sense, we call a $\Omega$-valued character of a toral algebra $\mathfrak{R}$ over $\mathfrak{F}$ an $\mathfrak{F}$-homomorphism of Lie $p$-algebras of $\mathfrak{R}$ into the extension $\Re$ of $\mathfrak{F}$. We then can prove

THEOREM 6. Let $\&$ be a (finite-dimensional) toral Lie p-algebra over $\mathfrak{F}$. Let $\Omega$ be an extension field of $\mathfrak{F}$. Then the following are equivalent:

(1) All characters of $\mathbb{R}$ with values in an extension of $\Omega$ are $\Omega$-valued;

(2) $\Omega_{\Omega}$ is isomorphic to a direct sum of copies of $\Omega$.

Such an extension $\Re$ will be called a splitting field for $\mathfrak{R}$; by appeal to (1) it is not hard to see that $\mathbb{R}$ has a finite splitting field, and indeed a unique minimal one within a given algebraic closure of $\mathfrak{F}$. This field is a galois extension of $\mathfrak{F}$.

TheORem 7. Let $\mathbb{2}$ be toral over $\mathfrak{F}$. Consider the properties:

(a) the only $\mathfrak{F}$-valued character of $\mathbb{R}$ is zero;

(b) \& contains no subalgebra isomorphic to $\mathfrak{F}$.

If F is finite, (a) and (b) are equivalent. On the other hand, there exist fields $\mathfrak{F}$ of all characteristics $p>2$ and two-dimensional toral algebras over $\mathfrak{F}$ which violate each of the implications $(\mathrm{a}) \Rightarrow(\mathrm{b}),(\mathrm{b}) \Rightarrow(\mathrm{a})$. In these examples the field $\mathfrak{F}$ may be taken to be perfect. 
A toral algebra $\mathfrak{R}$ over $\mathfrak{F}$ is called anisotropic if condition (a) of Theorem 7 is satisfied, semisplit if $\mathfrak{R}$ has a composition series with factors isomorphic to $\mathfrak{F}$. A semisplit toral algebra need not be split, even over a finite field.

TheOREM 8. Let $\mathfrak{R}$ be toral over $\mathfrak{F}$. Then $\mathfrak{R}$ has a unique maximal anisotropic subalgebra $\mathfrak{A}$ and a unique maximal semisplit subalgebra $\mathfrak{S}$. If $\mathfrak{F}$ is finite, $\mathfrak{R}=\mathfrak{Q} \oplus \mathfrak{S}$. There exist examples, as in Theorem 7, where $\mathfrak{R}=\mathfrak{A}+\mathfrak{S}, \mathfrak{A} \cap \mathfrak{S} \neq 0$, and examples where $\mathfrak{A} \cap \mathfrak{S}=0$ but $\mathfrak{R} \neq \mathfrak{Y}+\mathfrak{S}$.

The results above are imperfectly analogous with some for algebraic tori [1], [4], [5]. The final two theorems relate toral algebras and algebraic tori:

THEOREM 9. Let $T$ be an algebraic torus defined over the field $\mathfrak{F}$ of characteristic $p \neq 0$. Let $\Omega$ be a minimal (separable) splitting field for $T$, (5) the Galois group of $\Re / \mathfrak{F}, \mathfrak{R}=\mathbb{R}(T)$ the Lie algebra of $T$. Then $X(\mathfrak{R})$, the character group (under addition) of $\mathfrak{R}$, is isomorphic with $X^{*}(T) / p X^{*}(T)$ as groups with (S) as operators $\left(X^{*}(T)=\right.$ character group of $T$ ). If $p \neq 2, \Omega$ is a minimal splitting field for $\mathfrak{R}$, so that $T$ is split if and only if $\mathfrak{R}(T)$ is. This assertion fails for $p=2$.

If $m=\operatorname{dim} T=\operatorname{dim} \mathfrak{R}(T)$, then $X^{*}(T)$ is isomorphic to the free abelian group $Z^{m}$; if $\mathbb{R}$ is toral of dimension $m, X(\mathbb{R})$ is an elementary $p$-group of order $p^{m}$. Thus the following is a converse to Theorem 9:

ThEOREM 10. Let $\mathfrak{R}$ be an m-dimensional toral algebra over $\mathfrak{F}$. Suppose there is a finite galois extension $\Omega$ of $\mathfrak{F}$ splitting $\mathfrak{R}$, an action of $\mathbb{B}=\mathbb{S}(\Omega / \mathfrak{F})$ on $Z^{m}$, and a $\left(\mathfrak{S}\right.$-homomorphism of $Z^{m}$ onto $X(\mathfrak{Q})$. Then there is an algebraic torus $T$ defined over $\mathfrak{F}$, split by $\Re$, such that $\&$ is isomorphic to $\mathfrak{R}(T)$.

Proofs of these results will appear elsewhere.

\section{REFERENCES}

1. A. Borel and J. Tits, Groupes rêductifs, Publ. Maths. Inst. Hautes Etudes Sci. 27 (1965), 55-151.

2. C. Chevalley, Théorie des groupes de Lie, II: Groupes algébriques, Hermann, Paris, 1951.

3. N. Jacobson, Lie Algebras, Interscience, New York, 1962.

4. T. Ono, Arithmetic of algebraic tori, Ann. of Math. 74 (1961), 101-139.

5. I. Satake, On the theory of reductive algebraic groups over a perfect field, J. Math. Soc. Japan 15 (1963), 210-235.

6. H. Zassenhaus, On trace bilinear forms on Lie algebras, Proc. Glasgow Math. Assoc. 4 (1959), 62-72.

YALE University 